\title{
Crashworthiness optimization of front rail structure using macro element method and evolutionary algorithm
}

\author{
Mariusz Pyrz $^{1}$ (D) $\cdot$ Marek Krzywoblocki $^{1}$
}

Received: 28 October 2018 / Revised: 20 January 2019 / Accepted: 13 February 2019 / Published online: 22 March 2019

(C) The Author(s) 2019

\begin{abstract}
In this paper, the research on crashworthiness optimization of thin-walled structures is presented. The model of "S"-shaped frame subjected to complex crush load is analyzed. The objective of the study is to determine the optimal dimensions of the space frame cross section so as to achieve the maximal energy absorption of the structure and to fulfill the design requirements related to the crushing force value and the geometry relationship. The Visual Crash Studio (VCS) software, based on the macro element methodology, is used to simulate the response of a thin-walled beam during the impact and to determine crashworthy parameters. The results present a very good correlation with the finite element calculations but can be obtained in a much shorter time. The VCS software was coupled to evolutionary algorithm developed to determine the best solution. The optimization procedures revealed the necessity of a new design criterion related to maximal bending moments in the structure. This formulation enabled to obtain very good results after short processing on a PC computer. The proposed approach may be successfully used at early stages of the crashworthiness analysis.
\end{abstract}

Keywords Crashworthiness $\cdot$ Energy absorbing structure $\cdot$ S-shaped frame $\cdot$ Macro element method $\cdot$ Optimization $\cdot$ Evolutionary algorithm

\section{Introduction}

The vehicle components that create a structural frame are designed to respect a number of requirements simultaneously. There are several issues related to the production process and its economic aspects. On the other hand, there are some prerequisites to fulfill that are directly related to mechanical properties and safety standards. The crashworthy design of a vehicle structure has become the crucial task for decades in the automotive industry (Ambrosio 2001; Duddeck et al. 2016; Jones and Wierzbicki 1983; Christensen and Bastien 2015; Christensen et al. 2012).

Responsible Editor: Ren-Jye Yang

Mariusz Pyrz

mariusz.pyrz@pw.edu.pl

Marek Krzywoblocki

marek.krzywoblocki@gmail.com

1 Faculty of Automotive and Construction Machinery Engineering, Institute of Vehicles, Warsaw University of Technology, Narbutta 84, Warsaw, Poland
The development of a safe vehicle structure is a complex process where the variety of engineering tools is used to support works at each design stage. The technological progress that allows for the significant increase of calculation power gives us the possibility to introduce sophisticated numerical simulation methods in almost all domains related to the vehicle design, including also the optimization that efficiently tends to balance crashworthiness and light-weighting. The most commonly used tools in calculation of the crashworthy response are based on the finite element method (FEM). However, models developed for the FE analysis consist of a very large number of elements. In consequence, a highly detailed design representation of a vehicle influences the calculation time and computer clusters should be used to obtain the solution in a reasonably fast time. That feature of the FEM makes the structural optimization highly time-consuming and generally impossible to carry out on a standard PC. Moreover, the proper design of vehicle energy absorbing components requires the consideration of various approaches to find the optimal solution. However, the starting point for crushing response calculation can be a simple model (thereafter its complexity can be increased). That approach minimizes the risk of design mistakes committed at early design stages which may 
generate the costs when the overall design process progresses. Such an alternative is the application of the macro element method (MEM) well suited to crashworthiness simulations and characterized by the calculation time reduced tremendously with respect to FEA without affecting the overall results (Abramowicz 2004). The simplified approach for modeling large deformations of thin-walled structures was developed by T. Wierzbicki, W. Abramowicz, N. Jones, and others in the late 80 s of the last century (Wierzbicki et al. 1989; Wierzbicki and Abramowicz 1984; Jones and Abramowicz 1986). Subsequently, a formulation of the MEM was introduced by W. Abramowicz in the form of computerized versions (Impact Design Europe 2017). Very fast calculation time is the advantage of the MEM, and the computation of crashworthy parameters for quite complex structures usually takes up to several minutes on a standard PC. That fact gives us the possibility of introducing the optimization procedures into the vehicle development process.

The number of studies has been devoted to the optimal design and the calculation of crashworthy parameters of the front vehicle structure. One of the first investigations were carried out by $\mathrm{Ni}(1973,1974)$ who described the calculation procedures and relations between the global and local deformation of frames. The geometrical complexity of the front frame structure becomes the problem in an effortless prediction of crashworthy parameters. In case of a vehicle accident, the frame is exposed to axial, biaxial bending, and torsion load. That subject in the past was investigated by Meadows et al. (1992) and Chung et al. (1996). The study of crushing behavior of thin-walled structures was also carried out by Kim and Wierzbicki (2000). Their work started with the derivation of the analytical solution to collapse mechanism of thinwalled structures subjected to complex loading conditions (Kim and Wierzbicki 2001a). At the same time, Chen and Wierzbicki (2000) investigated the problem of torsional collapse. Subsequently, the works by Kim and Wierzbicki focused on the definition of the mathematical description of three-dimensional S-frames. The proposed calculation schemes converged with FEM simulation results as it was published in (Kim and Wierzbicki 2004). Additionally, the same authors carried out a study on the influence of different design aspects on the energy absorption related to the structure's weight (Kim and Wierzbicki 2001b). The publication presents the results of several cross-section configurations and characterizes proper design steps to achieve the crashworthy and the weight efficient design. A number of design approaches to the S-frame structures were presented by Hosseini-Tehrani and Nikahd (2006). The implementation of various cross-sectional orientation and a combination of steel and aluminum materials was studied to assess the best model configuration. The application of different materials within a single subassembly was a design strategy that had improved crashworthiness and reduced the mass of the structure.
In the recent time, several optimization procedures of the front rail structures were proposed. The research by Zhang et al. (2007) examined the response surface method (RSM) combined with design of experiment (DOE) to derive the relation between crashworthy and design parameters. Khakhali et al. (2010) proposed the multi-objective optimization solution of the rectangular cross-section S-frame exposed to axial impact. Authors used FEM to calculate the variety of simplified design scenarios of the front rail structure. Moreover, they considered the application of calculation scheme based on the evolved group method of data handling (GMDH) and neural networks to determine dependencies between the energy absorbed by the structure, the maximal force generated in the crush event, and design variables. The models obtained this way were processed by the genetic algorithm. The results presented a successful application of the described solution and gave useful conclusions to the optimization approach. De Gaetano et al. (2015) presented the idea of a vehicle's FEM modeling concept coupled with gradient-based optimization procedures. Authors highlighted the difficulties apparent in the optimization problem definition which resulted from the amount of design variables and the conflicting objectives to fulfill. The results showed the sensitivities of applied methods with respect to obtained solutions and indicate influence on calculation time. The recent review article (Fang et al. 2017) provides the state of the art related to the recent studies on design optimization for structural crashworthiness and energy absorption. It presents numerous research papers classified on the basis of design criteria, solution methods, optimization strategies, energy absorbing structures, and type of application. However, among the referenced publications, there is no position concerning the use of the macro element method in the optimization of crashworthy structures. The present paper and the previous work of authors (Pyrz and Krzywoblocki 2017) intends to fill this gap.

An extensive research on simplifying the mathematical description of frame's bending resistance crushing mechanics was performed by Y. Liu and M.L. Day and published in (Liu 2010a; Liu and Day 2006; Liu and Day 2007). Authors investigated several cases that involve circular, hexagonal, box, and channel cross sections. Additionally, the subject close to the present study was discussed earlier by Liu (2010b). The author investigated an S-shaped frame crushing behavior and linked it to optimal cross-sectional dimensions. The FEM was used to conduct crashworthiness analysis and to build the surface response function in order to maximize the specific energy absorption (SEA). Another instance of an S-shaped rail research was performed by Zhou et al. (2011). The work covered the investigation of the impact of different parameters on the crushing performance of vehicle components. The influence of different materials and cross-sectional dimension 
configurations was examined by means of orthogonal experiment method. The multi-objective problem was solved where the peak force level, SEA, assembly mass, and the specific total efficiency (STE) were considered as the objectives. The subject of the S-shaped frame optimization was also touched by Nguyen et al. (2014). Authors focused on the optimal design of reinforced rails. The multi-objective optimization process coupled with multi-objective particle swarm optimization algorithm (MOPSO) and RSM was employed to find the solution that fulfills the criteria imposed to the SEA and the peak force values. The authors indicated the advantages of the proposed optimization method and pointed out the potential to the application of reinforced S-shaped frames in practice. The example of S-frame optimization for crash events was also studied by Liu et al. (2014) who investigated the crashworthiness of various cross-sectional shapes (e.g., rectangular polygonal, non-convex polygonal, and multi-cell). The optimization procedures based on sequential response surface method (SRSM) showed the advantages of the internal reinforcement application. A more recent publication by Cai and Wang (2017) presents a parametric approach for S-shaped frame design and the implementation of the gray rational analysis (GRA). Simultaneously, for the same problem, alternative optimization methods were used (e.g., NSGA-II or MOPSO). The extensive works indicated that GRA was more effective comparing to other methods and returned the result with a better crash performance.

In this work, the problem related to the development of crashworthy structures and design of energy absorption elements is investigated. The cross-sectional dimensions of the S-shaped frame are determined using dedicated optimization procedures combined with the VCS software. The purposes of this study are to examine the efficacy of the presented solution and to obtain the structure that fulfills imposed design requirements and simultaneously returns the highest energy absorption level. The formulation of the optimization problem revealed the necessity of the application of a new design criterion, strictly related to the maximal bending moment in the analyzed structure. It enabled us to obtain very good results. This constraint was not mentioned in the literature before (for example in the very complete review paper by Fang et al. 2017). One of the advantages of the present approach is a very short calculation time of crash analysis (incomparably small compared to analogous FE study). Additionally, the optimization procedure provides directly the solution, related to the applied macro element model. The result does not need further verification (like for example the instance of RSM, searching the solution on the approximated function) or confirmation (like simplified CAE models, difficult to create and calibrate in the problem under consideration).

\section{Problem formulation}

The crushing behavior of the space frame (Fig. 1) subjected to quasi-static axial load is under consideration. The structure is built of 3 sections of length $S 1, S 2$, and $S 3$. Section 1 and section 3 are perpendicular to the axis of the applied load and are interconnected with section 2 that forms an angle $\varphi$ to section 1 and section 2 . This assembly is often applied in modern cars.

The problem concerns the optimization of cross-sectional dimensions of the space frame in order to achieve the maximal energy absorption of the structure. The frame is subjected to the load of profile presented in Fig. 2. Due to the specification of the load application, the dynamic and inertia effects are not taken into account within the simulation conditions.

A single hat cross-section frame is considered (Fig. 3), where the position of the local axis corresponds to a neutral axis of the beam. The geometry of the cross section is defined by the thickness $T 1$ and the thickness $T 2$. Moreover, crosssectional length parameters are denoted as $L 1$ and $L 2$, while the $L 2$ parameter belongs to two oblique walls of the hat cross section. The investigated cross section has implemented a reinforcement which thickness is not considered as a variable. However, the width of the reinforcement $W$ follows change of the variable $L 2$.

Fig. 1 S-shaped frame

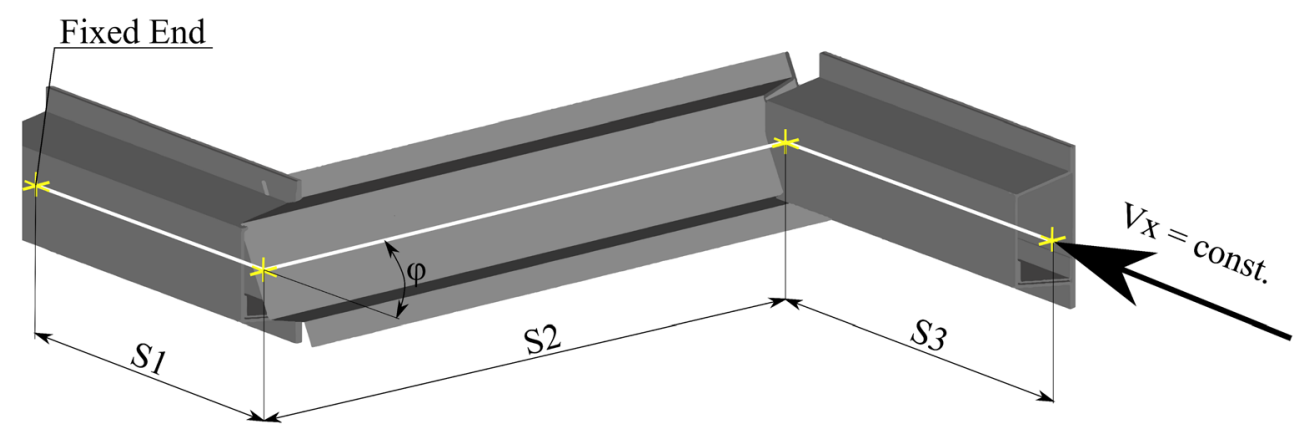


To find the most adequate solution, the specific energy absorption (SEA) parameter which represents the total energy absorbed per total mass of the structure is calculated. The final solution is influenced by a number of factors which characterize the crashworthy structure. In the event of a crash, a save design of the structure ensures the survive conditions for vehicle's occupants. That means the level of maximal crushing force $P_{\max }$ must be maintained below the critical values. Thus, the best solution is selected basing on the critical value of maximal force definition, denoted as $P_{\text {max-ref }}$. An additional limitation was imposed on the mean crushing force $P_{m}$ as an example of design constraint which in practice collides with the main objective condition for maximization of absorbed energy. Furthermore, additional dimension constraints were imposed on the resulting design. It was assumed that the thickness $T$ of crosssection components are kept within the range declared by $T_{\min }$ and $T_{\max }$. Also, the length $L$ of the cross-section side walls are placed between lower $L_{\min }$ and upper $L_{\max }$ limits. It was assumed also that the relation between the summary wall lengths and their summary thicknesses is limited to the range with the lower bound $(L / T)_{\min }$ and the upper bound $(L / T)_{\max }$, which reflects the definition of a thin-walled cross section that is less sensitive to the premature global bending (Jones and Abramowicz 1986).

The resulting optimization problem can be formulated as follows:

determine the dimensions $T 1, T 2, L 1$, and $L 2$ of the cross section so as to maximize the SEA

$\operatorname{SEA}(T, L) \rightarrow \max$

Fig. 2 Velocity profile and to satisfy the following constraints:

$P_{m}(T, L) \leq P_{m-r e f}$

$P_{\max }(T, L) \leq P_{\text {max }- \text { ref }}$

$T_{\min } \leq T \leq T_{\max }$

$L_{\min } \leq L \leq L_{\max }$

$$
(L / T)_{\min } \leq L / T \leq(L / T)_{\max }
$$

The solution of the problem (1)-(6) presented by authors in their previous publication (Pyrz and Krzywoblocki 2017) and devoted to the optimization of straight thin-walled tubes generally lead to very satisfactory results. However, preliminary study related to the S-shaped frame under consideration showed the necessity of additional conditions. As the space frame deformation involves bending phenomena, the limitations to maximal bending moments were defined. That situation refers to a practical design scenario of the S-frame in which individually controlled deformation zones are activated sequentially. The solution minimizes the risk of an excessive peak force development. On the other hand, the maximal bending moment should be high enough to ensure the sufficient support for deformation of the front vehicle components acting mainly in the progressive axial crush, and to prevent large intrusions to the passenger compartment. Thus, the following optimization constraints are proposed to be included in the problem formulation:

$$
\left|M_{x}(T, L)\right| \leq M_{x-\max -r e f}
$$

$\left|M_{y}(T, L)\right| \leq M_{y-\max -r e f}$

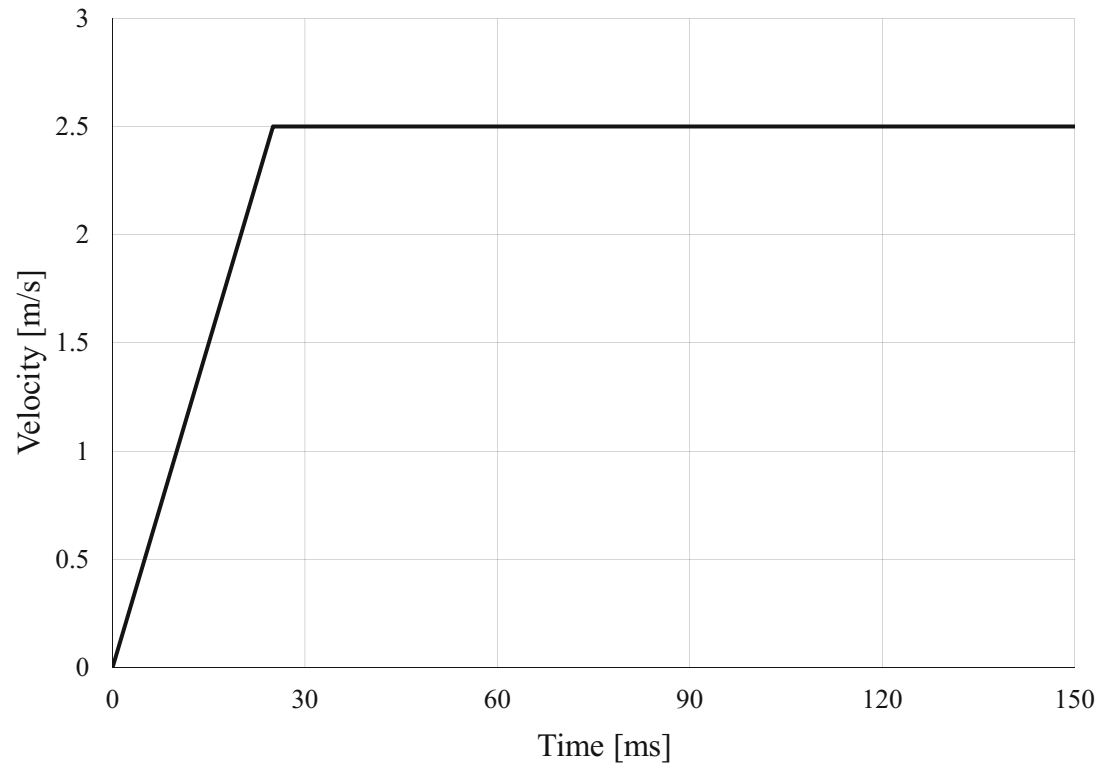




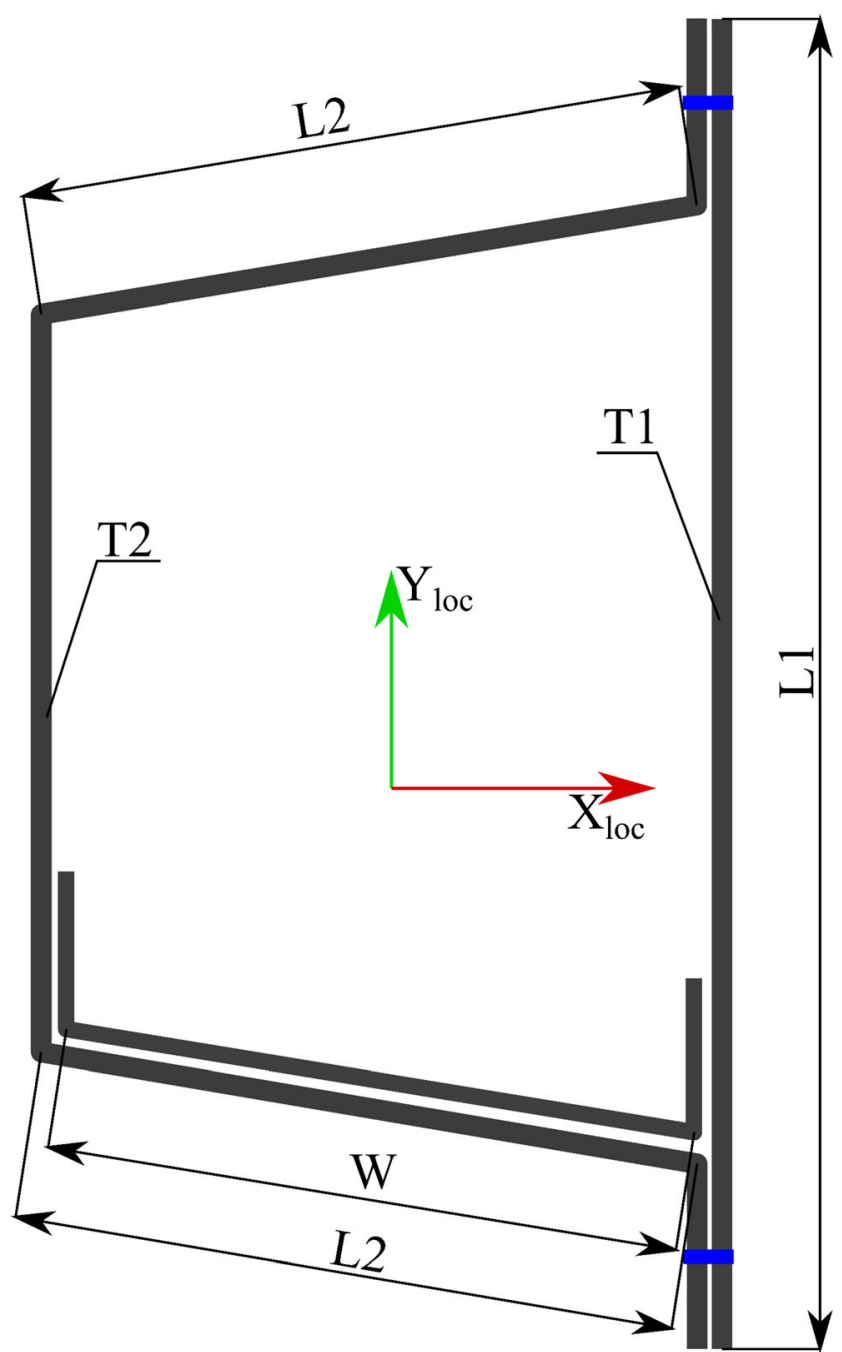

Fig. 3 Variables of a single hat cross section

where the maximal bending moments $M_{x-\text { max-ref }}$ and $M_{y \text {-max-ref }}$ represent the upper limits for bending in the local $\mathrm{X}$ and local $\mathrm{Y}$ directions (Fig. 3) of the frame section.

Finally, another additional condition is suggested:

$d M=\left(M_{x-\max -r e f}-\left|M_{x}(T, L)\right|\right)^{2}+\left(M_{y-\max -r e f}-\left|M_{y}(T, L)\right|\right)^{2} \rightarrow \min$

The variable $d M$ in (9) defines the "distance" to the desired bending moment. It suggests that the investigated space frame is prone to collapse with possibly maximal bending moment allowed. The combination of the imposed conditions leads to the maximization of energy absorbed by the structure, repeatability, and consistency of results. Without (9), the problem was not constrained enough to produce a unique solution. This situation will be illustrated by the numerical examples presented in chapter 5 .

\section{Modeling methods}

\subsection{Macro element method and Visual Crash Studio}

The assessment of structural crashworthiness has been one of the most challenging and responsible tasks in the vehicle development process. Commonly used FEM introduces a variety of advantages. However, attempts of the FEM application in optimization procedures become non-efficient. Therefore, alternative methods emerged to face the problem of a long calculation time of crashworthy parameters. One of them is the macro element method (MEM) developed by T. Wierzbicki, W. Abramowicz, and N. Jones. The methodology uses a mathematical description of large deformation of thinwalled structures which was developed basing on trial deformation functions (Abramowicz 2004). The analytical foundations are derived from the kinematic method of plasticity and energy method of classic elasticity. But instead of calculating the general deformation from classic equations of equilibrium, it assumes the specific kinematics. Thus, a typical buckling or folding pattern in folded structural elements is isolated, and its mechanical response is described. The core of MEM method is the definition of the super folding element (SFE). The SFE represents energy absorption mechanisms of a corner line of a prismatic beam. It is a counterpart of a single folding wave of a deformed column presented in Fig. 4.

The macro element method is implemented in the form of a calculation processor within the Visual Crash Studio software (VCS) developed by Impact Design Europe company. It allows for assessment of crashworthy parameters with much lower processing effort comparing to FEM solvers. The VCS is a CAD environment that calculates crushing parameters of prismatic beams in two stages. The first one is discretization of beam's cross section into SFE. The geometrical and material properties of the cross section are used for the initial calculation of crashworthy parameters. Subsequently, the returned data are used to construct the space model consisted of super beam elements (SBE). The developed model, composed of prismatic beams, is limited to a certain type of deformation. It means that the deformation of all models that have been described in this article progresses always in the same way. Several benchmark tests conducted by Takada and Abramowicz (2003) showed that MEM needs an incomparably shorter pre-processing time comparing to FEM (i.e., the calculation effort is much lower), and the resulting results present a high accuracy. The most recent assessment of MEM application in the crashworthiness analysis was conducted by Georgiou and Zeguer (2018). Their work investigates advantages and weak points of simplified crash phenomena modeling using MEM. In industrial practice, the energy absorbing structural elements are properly triggered so as to enable the progressive collapse and to guarantee proper 
Fig. 4 Bent prismatic column and super folding element

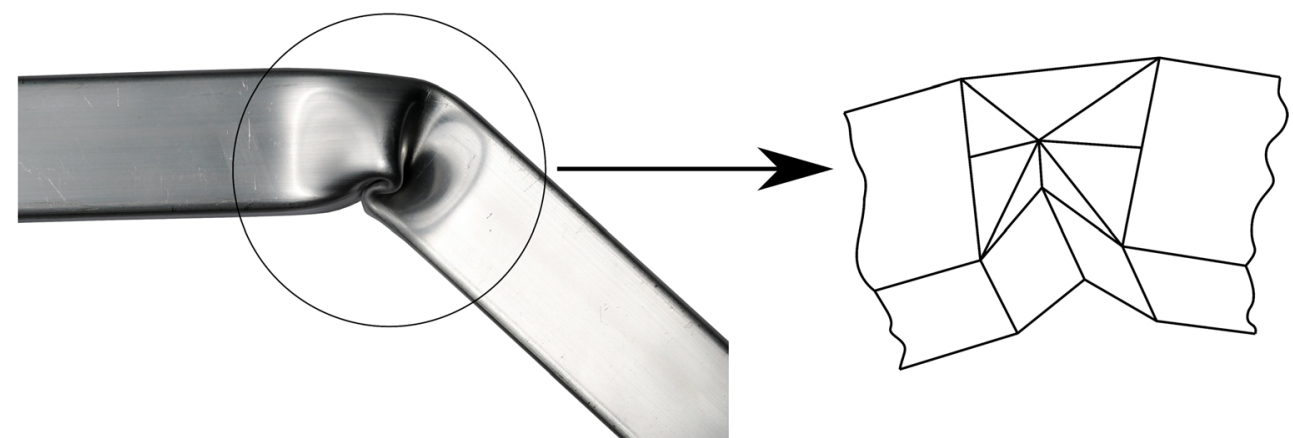

folding without internal contacts. This behavior is precisely predicted by VCS even for complex structures.

\subsection{Validation of macro element method}

A number of papers which describe the crushing behavior of the S-shaped frames were mentioned in chapter 1. In order to validate the MEM procedure, the study presented by Kim and Wierzbicki (2004) was used as a reference. The initial configuration of the model was validated using the data set provided in (Kim and Wierzbicki 2004). The dimensions of the 3D Sframe chosen for validation are represented by three length values $S 1=330 \mathrm{~mm}, S 2=490 \mathrm{~mm}, S 3=330 \mathrm{~mm}$ and angle $\varphi=30$ degrees. The angle determines eccentricities of the individual sections $\mathrm{S}$ of the $\mathrm{S}$-frame. The $\mathrm{S}$-frame has a rectangular cross section $80 \times 80 \times 2.4(\mathrm{~mm})$, where $80 \mathrm{~mm}$ is the sides' length and $2.4 \mathrm{~mm}$ is the cross-section thickness. The material used in the simulation is an aluminum alloy. Therefore, strain rate effects are not considered in comparison. The quasi-static load condition is assumed. One end of the Sframe has been assigned the velocity like it is presented in Fig. 2, the opposite end is clamped. The simulation output was the axial force measured at the moving end of the structure. Figure 5 presents the results where curves obtained from simulation using Visual Crash Studio and Pamcrash (FEM) software are compared to the theoretical derivation by authors of the referenced article (Kim and Wierzbicki 2004). It can be noticed that all three curves represent a very good correlation of results. The numerical examples presented in the article refer to non-symmetric geometrical cases. However, there are factors that influence the kinematics of crushing simultaneously maintaining similar crushing mode for each generated model. First is the fact that the hat component is the major segment of the cross section. Second, the constraint (6) promotes the cross section's uniformity.

\section{Optimization procedure}

\subsection{Evolutionary algorithm}

Evolutionary algorithms (EA) are general purpose, stochastic search methods inspired by natural evolution, hereditary, and survival of the fittest individual (Michalewicz 1996; Eiben and Smith 2003). They are naturally adapted to solve unconstrained optimization problems and are able to deal with
Fig. 5 S-frame crushing response - validation example

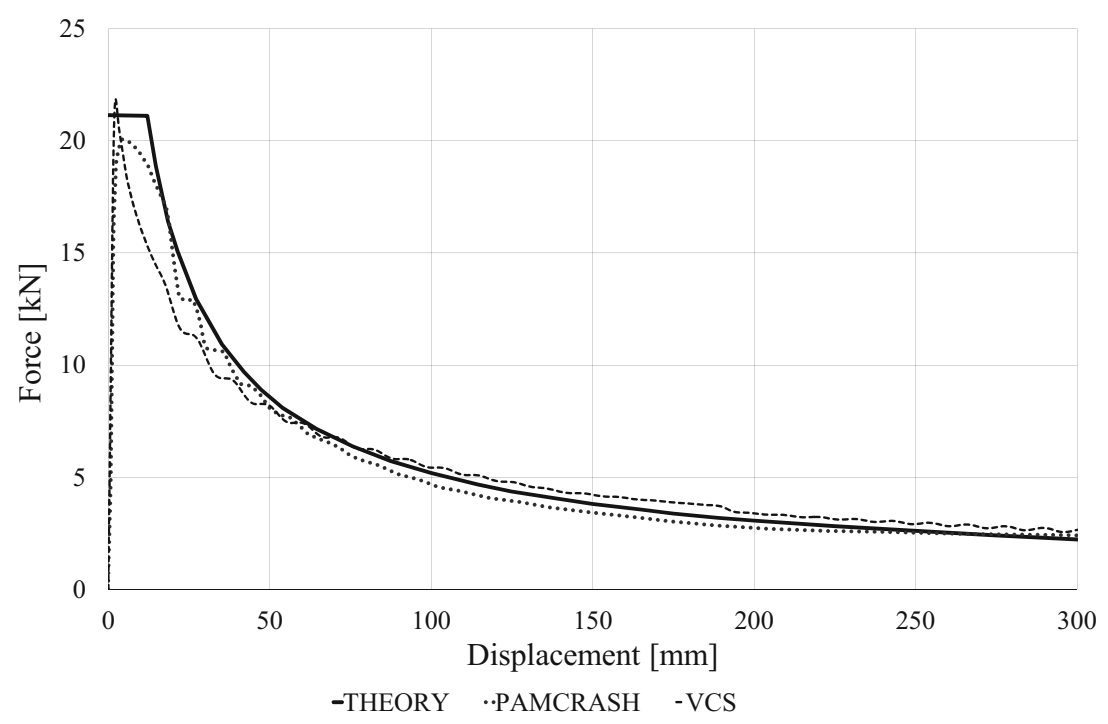


difficult, big size, high cardinality, and multi-modal problems. They have been applied with success to a large variety of optimization problems and became a recognized solving methodology despite its random character and relatively high computing time.

The EA manipulate an encoded representation of the problem and emulate evolutionary theories using adapted genetic operators. The evolution is simulated using a set of biologically inspired operators (like selection, crossover, mutation) and random parameters. In this process, better individuals have more chances to survive and to form next generations. New designs are usually better, and they replace members of old populations and this way the evolutionary method converges. The algorithm processes at a time a fixed number of potential solutions, performing an efficient multi-directional exploration and exploitation of the design space. The process continues for several iterations, and the solution is given by the best-found design, characterized by the best fitness (usually maximal) - a value that measures how good is the proposition of the solution. The EA has been popularized by genetic algorithms, which used standard binary string encoding of design variables. The evolution algorithms promote different data structures and corresponding adapted operators. All these search procedures apply the similar evolution scheme represented in Fig. 6.

The steps of the flowchart depicted in Fig. 6 were coded in an independent computer program, developed by authors in $\mathrm{C \#}$ programming language. Each stage of the algorithm is treated as an independent operation block which calls the input data as arguments at the start and returns the output in the form of the processed group of individuals.

\subsection{Formulation of the adaptation function}

The solution of the optimization problem formulated in the chapter 2 demands the appropriate definition of the adaptation function that measures the quality of the result. In the present study, the penalty function approach has been used in order to take into account the objective and the constraints. The calculations started with the initial formulation using the "classical" objective and constraints as the studies presented in the "state of the art" paper by (Fang et al. 2017). That standard formulation turned out to be not very efficient. Thus, it was expanded by two additional terms related to the bending moment. The final form of fitness function applied in this study defined as.

$$
\begin{aligned}
& F_{F i t}=\frac{S E A(L, T)}{S E A_{r e f}}-F_{P 1}\left(P_{m}\right)-F_{P 2}\left(P_{\max }\right)-F_{P 3}(L, T)+ \\
& -F_{P 4}\left(M_{\max }\right)-F_{P 5}(d M)
\end{aligned}
$$

The fitness function (10) consists of several components. The main component of $F_{F i t}$ corresponds to the objective function $S E A$ (1), divided by the reference value $S E A_{\text {ref }}$ in order to keep this value within an appropriate range. The $S E A_{\text {ref }}$ value is determined in a trial run of the processing algorithm. The consecutive components are the constraints from the problem definition $(2-3)$ and (6-9) included as the penalty functions. Thereby, the penalty components are responsible for decrease the overall value of $F_{F i t}$ when the calculated solution returns the result that violates the defined range of constraints.

The first penalty component $F_{p l}$ is responsible for the penalization linked to the mean crushing force $P_{m}$ (2). When $P_{m}$ of the individual is above $P_{m-r e f}$ then the $F_{F i t}$ value is decreased. The corresponding $F_{p l}$ term is defined as follows:

$$
F_{P 1}=\operatorname{coef}_{P m} \max \left(0, \frac{P_{m}-P_{m-r e f}}{P_{m-r e f}}\right)
$$

The factor $\operatorname{coef}_{P m}$ represents the penalty coefficient. In the numerical examples, coef $_{P m}$ value has been set to $0.25^{*} c-r e f$ to keep the right proportions between the components of fitness function, where $c-r e f$ is equal to the main component of the fitness function $c-r e f=S E A / S E A_{\text {ref }}$.
Fig. 6 A general formulation of evolutionary algorithm

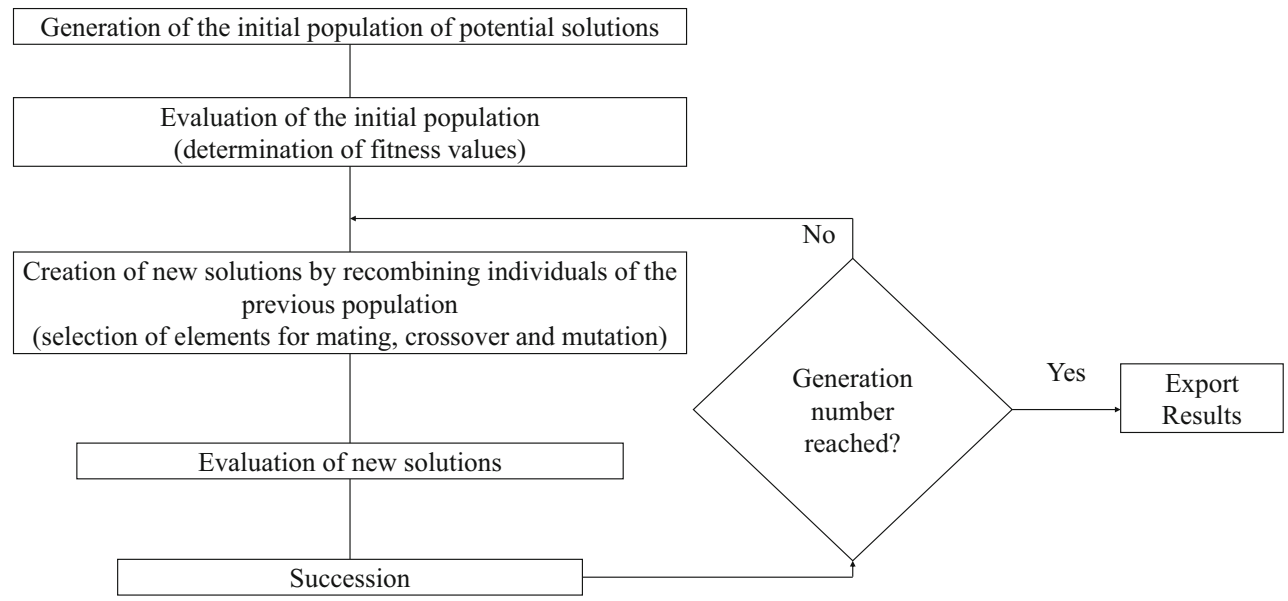


The penalty component $\mathrm{F}_{P 2}$ is related to the maximal force $P_{\text {max-ref }}$ constraint (3) and may be written as:

$F_{P 2}=\operatorname{coef}_{P \max } \max \left(0, \frac{P_{\max }-P_{\max -r e f}}{P_{\max -\text { ref }}}\right)$

Analogically to the previous penalty component, the $F_{P 2}$ decreases the fitness function value when the actual $P_{\max }$ is higher than the maximal force allowed. In this study, the penalty coefficient $\operatorname{coef}_{P \max }$ is set to $0.2 * c-$ ref.

The next component $F_{P 3}$ is responsible for the decrease of the fitness function value when the ratio $L / T$ goes beyond the allowable range located between $(L / T)_{\min }$ and $(L / T)_{\max }$. In this study, the lower and the upper limit have been set respectively to 20 and 50, so the solution must satisfy the constraint (6) in the form $L / T \in(20,50)$. The chosen values correspond to dimensional proportions of cross section which can be treated as a thin-walled structure that deforms in a predictable way. The condition is implemented for the lower and the upper bounds separately and may be written

$F_{P 3}=\operatorname{coef}_{L T}\left|\max \left(0, \frac{L / T-(L / T)_{\max }}{(L / T)_{\max }}\right)+\min \left(0, \frac{L / T-(L / T)_{\min }}{(L / T)_{\min }}\right)\right|$

The $\operatorname{coef}_{L T}$ is the penalty coefficient, set to $0.3 * c-r e f$.

The $F_{P 4}$ is responsible for the decrease of the fitness function value when the maximal bending moments exceed the reference values $(7,8)$. It is formulated as:

$$
F_{P 4}=\operatorname{coef}_{M \max }\left(\begin{array}{l}
\max \left(0, \frac{M_{x}(T, L)-M_{x-\text { max }-r e f}}{M_{x-\text { max }-r e f}}\right) \\
\max \left(0, \frac{M_{y}(T, L)-M_{y-\max -r e f}}{M_{y-\max -r e f}}\right)
\end{array}\right)
$$

Similarly, to previous components, the coef $_{M \max }$ is the penalty coefficient, set to $0.2 * c-r e f$.

The last component of the fitness function, in fact, changes the problem formulation to multi-objective optimization task. The additional objective is implemented in form of penalty function expressed by the term $F_{P 5}$. The corresponding penalty function represents the normalized square difference of bending moments and is defined by the expression

$F_{P 5}=\operatorname{coef}_{d M}\left(\frac{\left(M_{x-\max -r e f}-\left|M_{x}(T, L)\right|\right)^{2}+\left(M_{y-\max -r e f}-\left|M_{y}(T, L)\right|\right)^{2}}{\left(M_{x-\max -r e f}\right)^{2}+\left(M_{y-\max -r e f}\right)^{2}}\right)$

The coef $_{d M}$ is the penalty coefficient with the value set to $1.5^{*} c-r e f$. The values of penalty coefficients were determined in the trial runs that investigated the sensitivity of each coefficient to the overall results. The implementation of two additional components (14)-(15) in the fitness function (10) goes beyond the "classic" formulation found in the optimization of thin-walled structures (Fang et al. 2017). It corresponds to the advantageous folding mode in the assumed crushing scenario modeled in VCS, promotes the primary deformation mechanism and facilitates finding the best solution.

The constraints (4) and (5) are taken into account directly in the EA program that generates parameters $T_{i}$ and $L_{i}$ randomly within the range defined by the lower and the upper limits and fixed by the user. The values of remaining components of the fitness function problem, e.g., (1-3) and (6-9) are determined on the basis of the VCS simulation results.

\subsection{Evolutionary operators}

The evolutionary algorithm developed for this study uses the floating point, natural representation of design variables. Parameters of the optimization problem are represented directly by a string of $N$ design variables, i.e., the parameters $T 1, T 2, L 1$, and $L 2$. Each individual corresponds to a S-shaped frame model which parameters are generated by the evolutionary algorithm. Thus, the "population" consists of several independent structures. The evaluation of the population at each iterative step requires the numerical determination of all necessary crashworthiness parameters by the VCS software.

The EA starts with the creation of the initial population. The defined number of S-frame models (the population) is generated. A dimensional specification of each model is set randomly respecting the range defined for each variable. Then, all models are sent to the VCS processor to calculate crashworthy parameters. Subsequently, the fitness value of each individual in the population is estimated.

When the initial population is created, the algorithm enters the main evolution loop (Fig. 6). It starts with the selection process based on the initial population. The objective of this stage is to collect chromosomes for mating. The selection procedure gives more chance to reproduce for individuals which are closer to defined environmental criteria. In the developed algorithm, the selection operator uses the tournament strategy. It draws each time a given number of models (value set to 4), compares them, and keeps the individual with the highest fitness function value. This way the selection of individuals equal to the initial population is performed.

The next operator used in the loop is the crossover. It enables mating between individuals and the creation of the offspring. The result of the procedure depends on the value of the randomly generated crossover probability. If this value is above the assumed crossover probability parameter $C P$, then parent chromosomes are taken without modification as new children. In the opposite case, parent chromosomes undergo crossing to produce children. In the proposed algorithm, two parent individuals are selected randomly. They exchange their values by taking the weighted average - the arithmetic recombination (Eiben and Smith 2003). 
The mutation operator is applied to introduce diversity in the population. Similarly, to the crossover operator, the mutation probability $M P$ is defined. The randomly generated mutation probability is compared with $M P$. The selected gene of the processed individual is substituted by a new random value when $M P$ is above the generated mutation probability.

The final operator within the evolution loop is succession. It selects the survivors that pass to the new population (trying to maintain the diversity of the resulting generation). In the proposed algorithm, the succession operator gathers the newly created "offspring" population, next sorts individuals with respect to the fitness value, and keeps percentage contribution of the best solutions. The same procedure is repeated for the previous "parent" generation. The new population is built from the survivors of two groups. In the presented algorithm, new populations are created by taking $40 \%$ of best individuals from the parent population and $60 \%$ from offspring population.

\subsection{Coupling to VCS program}

The communication between the optimization algorithm and the crush analysis is ensured through the interface developed in $\mathrm{C \#}$ programming language. The simplicity of the solution lies in an object-oriented programming paradigm. The recognition of specific data fields as individual objects facilitates data management and development of optimization tool. Therefore, it is easy to process automatically hundreds of VCS models. The optimization procedure starts from the definition of input data which consists of a geometry and material information, sets of design variables and other parameters necessary for VCS software. The EA reads the data, applies specific values related to design variables, and runs calculation of crashworthy parameters in VCS batch mode. The calculation results are collected from VCS output files via developed interface software and processed for each model individually according to the optimization scheme. These sequences of events are repeated within several loops of the simulated evolution as it was described in detail in the previous chapter.

\section{Numerical examples}

The presented numerical examples concern the S-shaped frame presented in the chapter 2 . They investigate different formulations of the optimization problem and analyze the efficiency of the applied method. The results are presented in Figs. 7, 8, 9, 10, 11, 12, 13, and 14 and Tables 2, 3, and 4. Three cases are compared and discussed.

\subsection{Space frame beam model}

The space frame model, build using macro element methodology, consists of one material, one cross section, four nodes, and three separate super beam elements (Fig. 1). The frame sections have the dimensions $S 1=330 \mathrm{~mm}, S 2=515.85 \mathrm{~mm}$, and $S 3=330 \mathrm{~mm}$. Section 1 and section 3 are perpendicular to the axis of the applied velocity and are interconnected with

\section{Fig. 7 Variable T1}

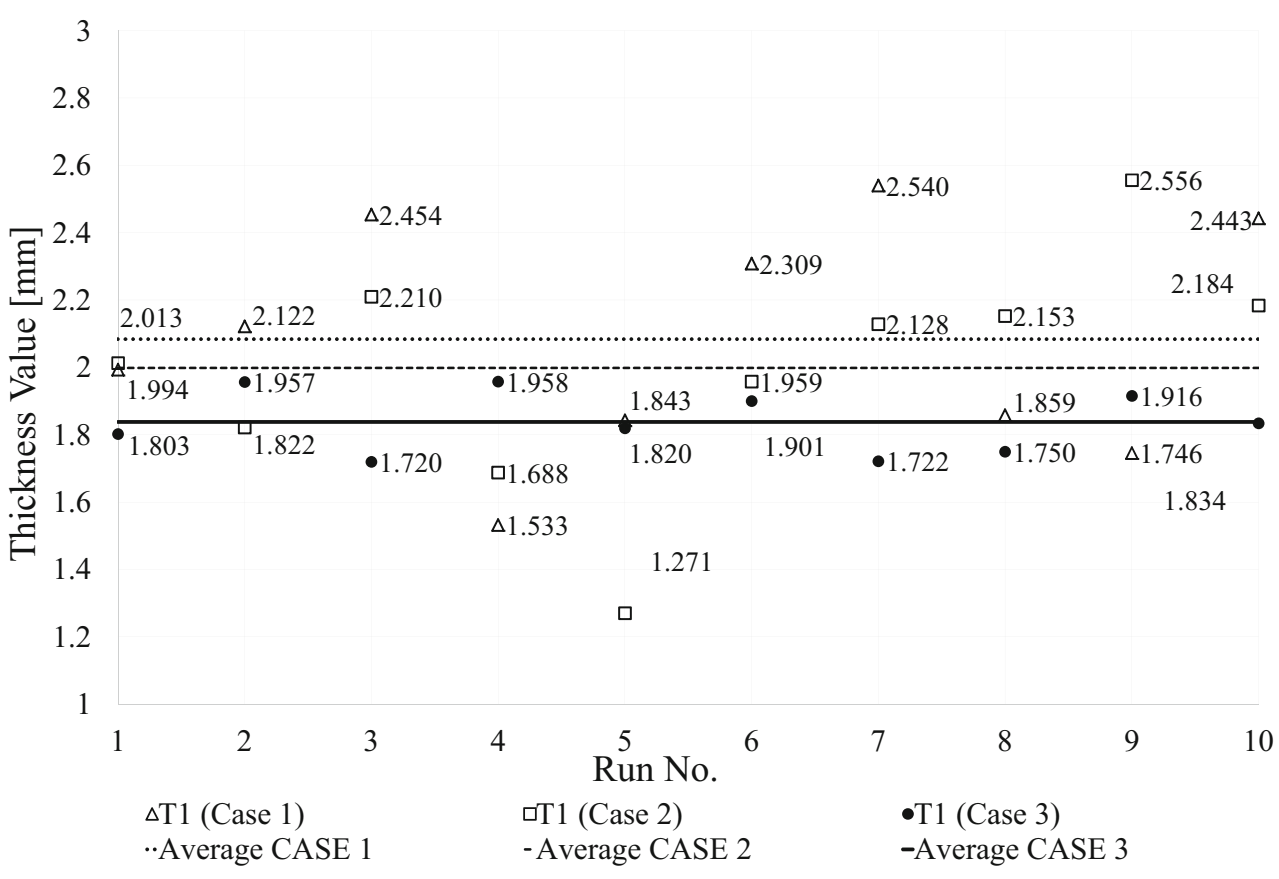


Fig. 8 Variable L1

要

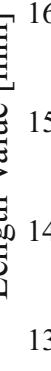

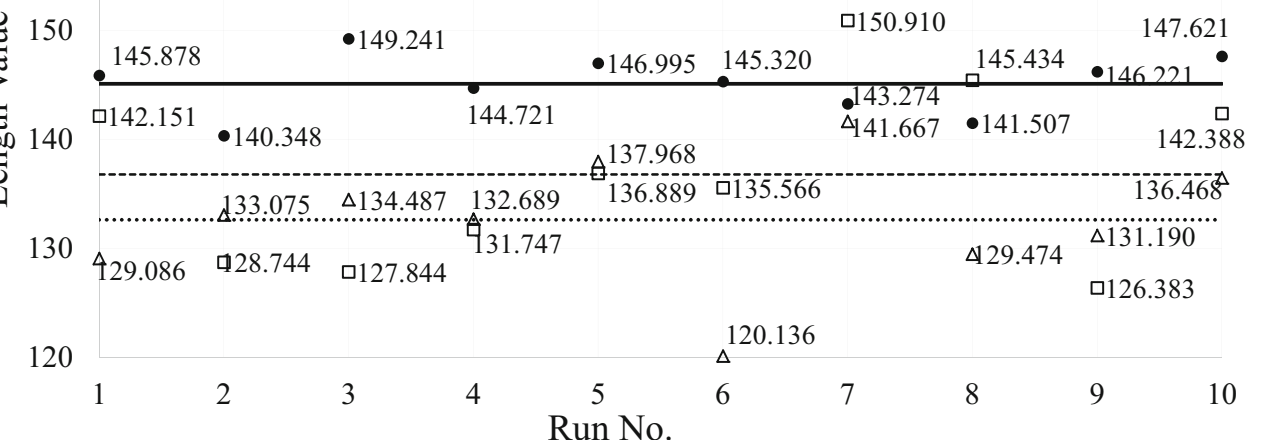

$\triangle \mathrm{L} 1$ (Case 1)

..Average CASE 1
口L1 (Case 2)

-Average CASE 2 $\bullet$ L1 (Case 3)

-Average CASE 3 section 2 that forms the angle $\varphi=30 \mathrm{deg}$ to section 1 and section 2 .

The thin-walled beam models with single hat cross sections are applied. The initial, reference dimensions are $T 1=T 2=$ $3 \mathrm{~mm}, L 1=180 \mathrm{~mm}$, and $L 2=90 \mathrm{~mm}$ (Fig. 3).

The calculation of crushing parameters is performed using the external solver of Visual Crash Studio software executed in the batch mode. The parameters of S-shaped frame models are summarized in Table 1.

\subsection{Initial optimization problem (case 1)}

First runs of the optimization algorithm were performed to study the results obtained for the initial formulation of the problem. In this case, only the constraint conditions described by (2) to (6) were taken into consideration. Since EA use intensively random numbers to generate the best solution, 10 independent runs of the optimization algorithm were carried out to study the efficiency of the

Fig. 9 Variable T2

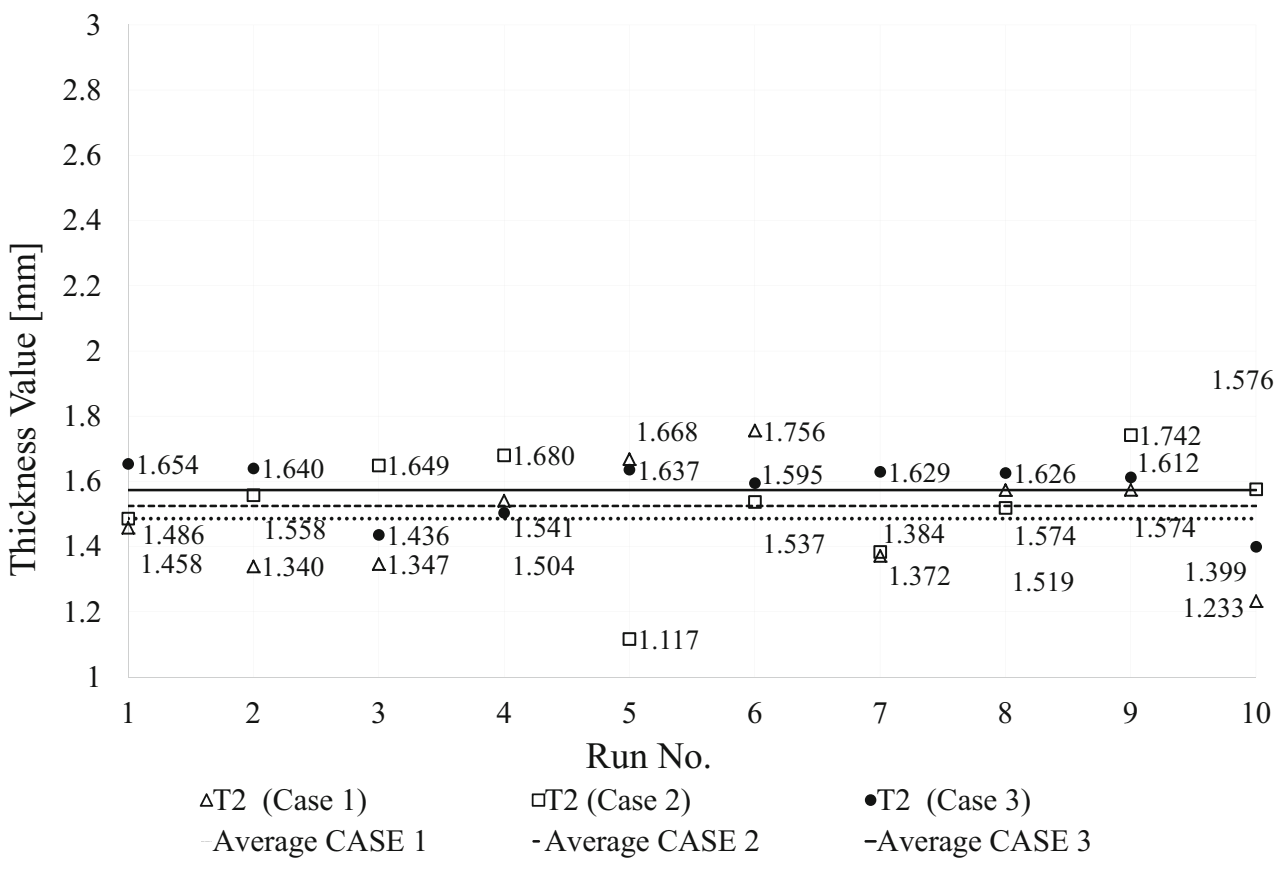


Fig. 10 Variable L2

90

85

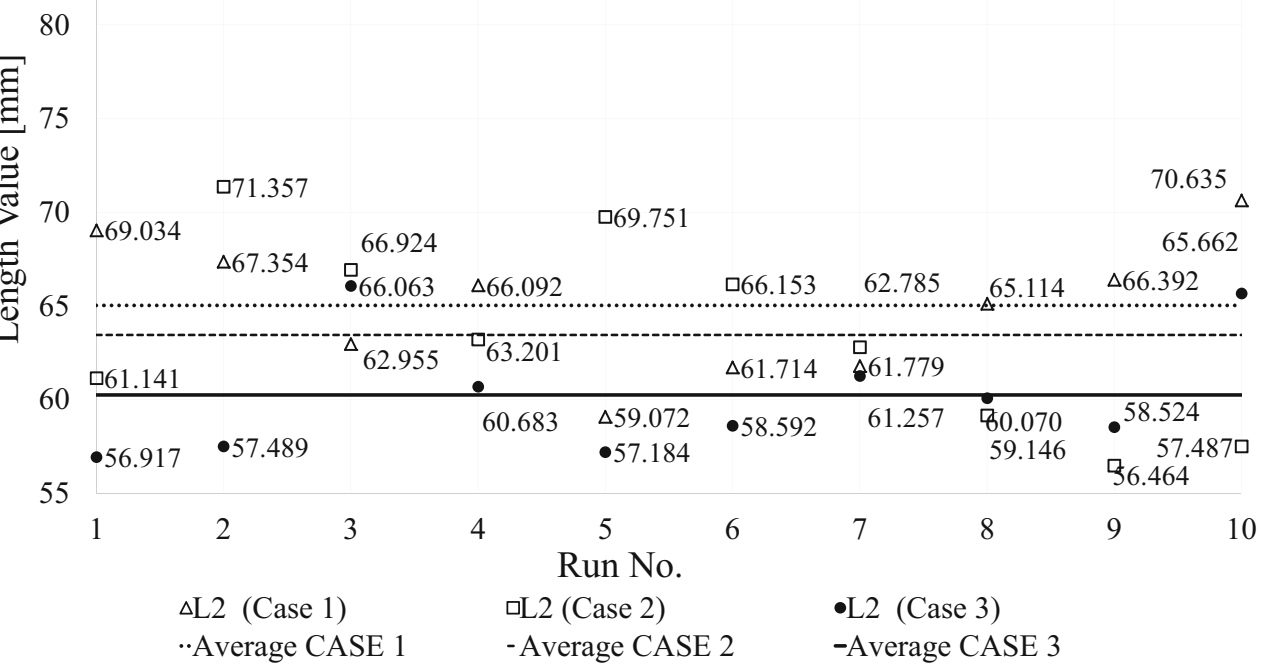

procedure. In Figs. 7, 8, 9, and 10, there can be noticed a large dispersion of all dimensions $T 1, T 2, L 1$, and $L 2$ which are treated as values to determine by EA. Such wide dispersion of results, especially for thickness variables, is not acceptable from the optimization point of view. Solutions obtained for case 1 have the average weight equal $7.59 \mathrm{~kg}$ which is $0.2 \mathrm{~kg}$ heavier than the result which returned the highest fitness value and $0.47 \mathrm{~kg}$ heavier than the lightest solution returned in 10 independent runs of the algorithm. The values of the minimum mass and the highest energy absorbed (which can be easily transformed into specific energy absorption) are not the only objective parameters. Therefore, the lightest solution is not the best one. The optimization variables corresponding to the result with the lowest mass are $L 1=131.190, L 2=66.392, T 1=1.746$, and $T 2=1.574$ (all expressed in millimeters). The value of the peak force is equal to $52.534 \mathrm{kN}$ which is close to the limit of $53 \mathrm{kN}$.
Fig. 11 Fitness function final values

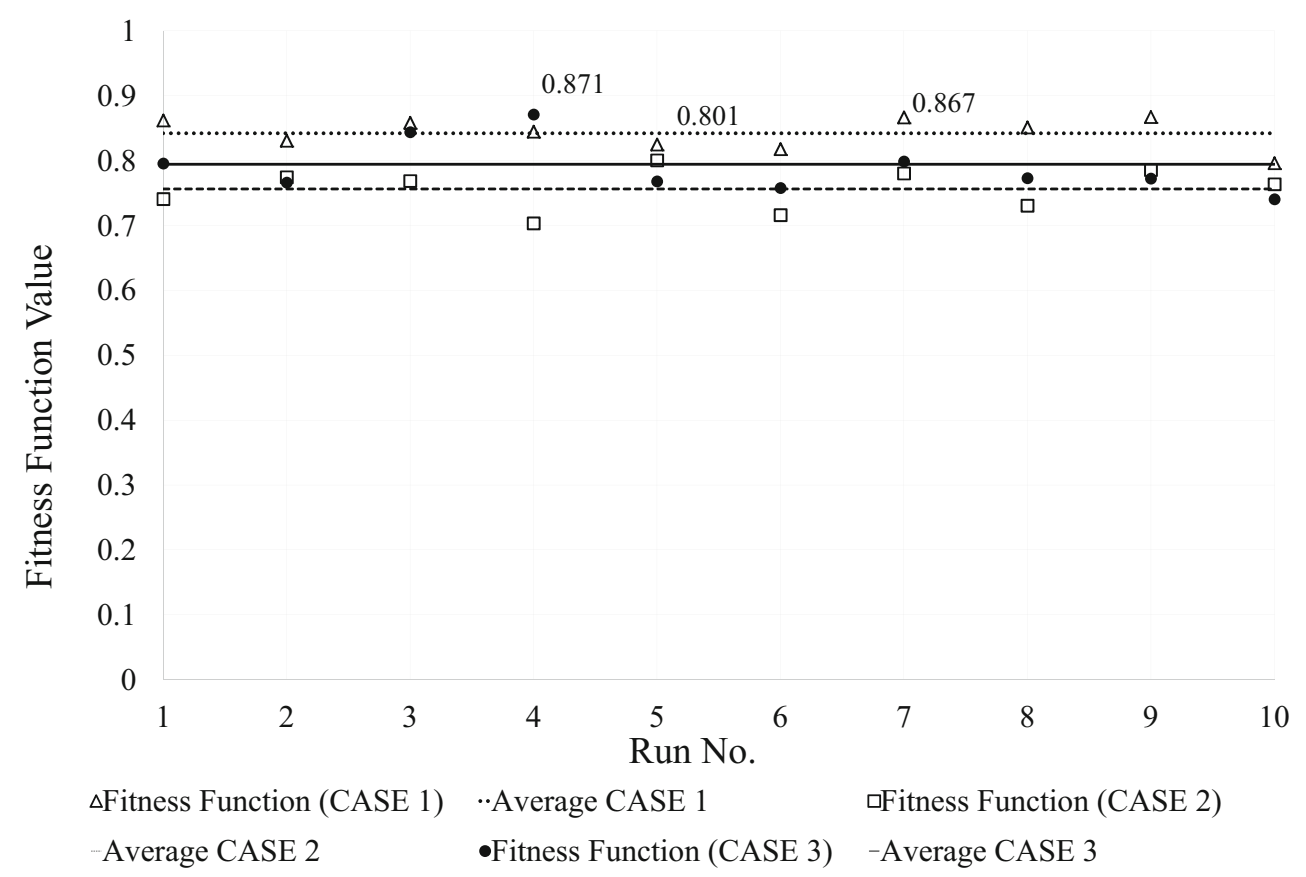


Fig. 12 Comparison of forces $[\mathrm{kN}]$ acting in $\mathrm{X}$ direction vs. $\mathrm{X}$ displacement $[\mathrm{mm}]$
60

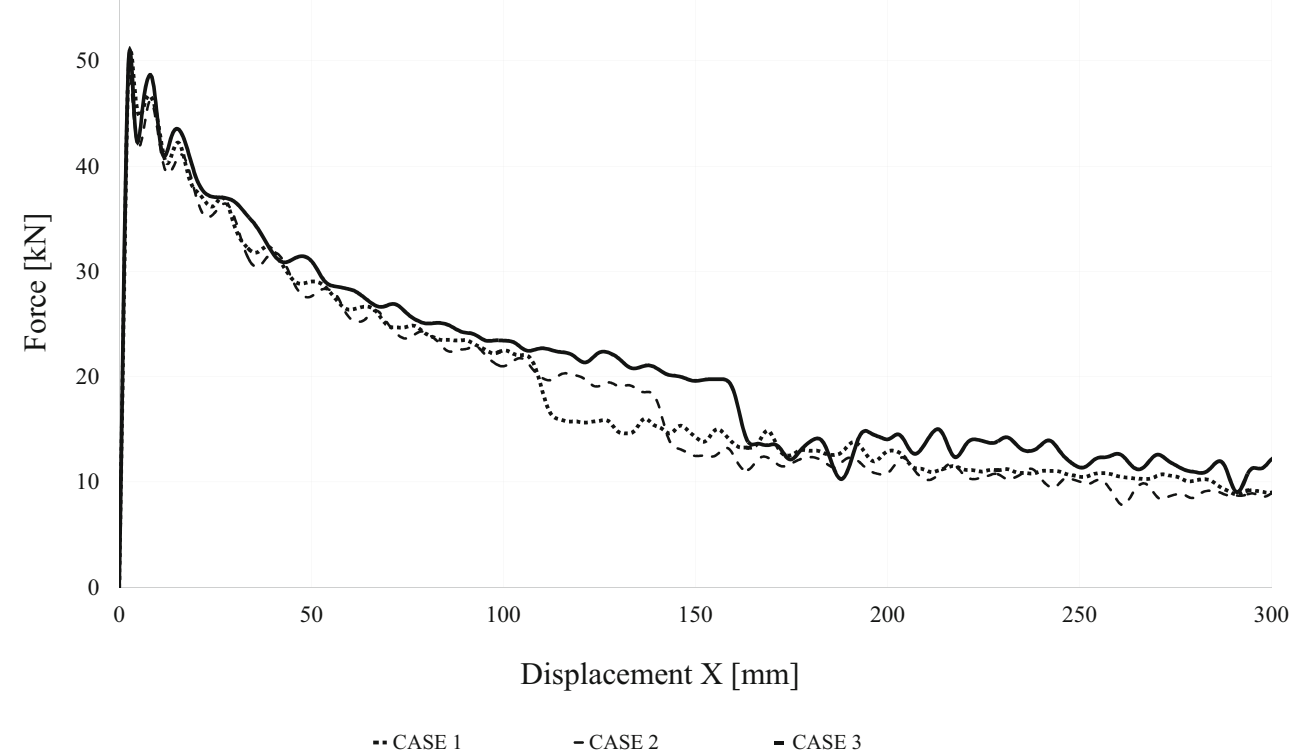

The mean crushing force is much below the assumed $P_{m \text { - }}$ ref equal to $25 \mathrm{kN}$. The actual mean force for the lightest result model is equal to $20.229 \mathrm{kN}$. The case 1 is represented by the highest average of fitness function value in Fig. 11. The analysis of the obtained results suggests that some effort must be devoted to finding a better solution. The initial problem formulation did not lead to expected, narrow range of result parameters in several runs of the optimization algorithm. The remaining cases present a successful way of the modification of the formulation of the problem.

\subsection{Modified problem formulation (case 2)}

The second approach complements the problem definition with additional constraints (7) and (8). The values presented in Figs. 7, 8, 9, and 10 do not show better convergence. The relation between average values of variables and their stan-
Fig. 13 Comparison of energy absorbed by the space frame for three cases

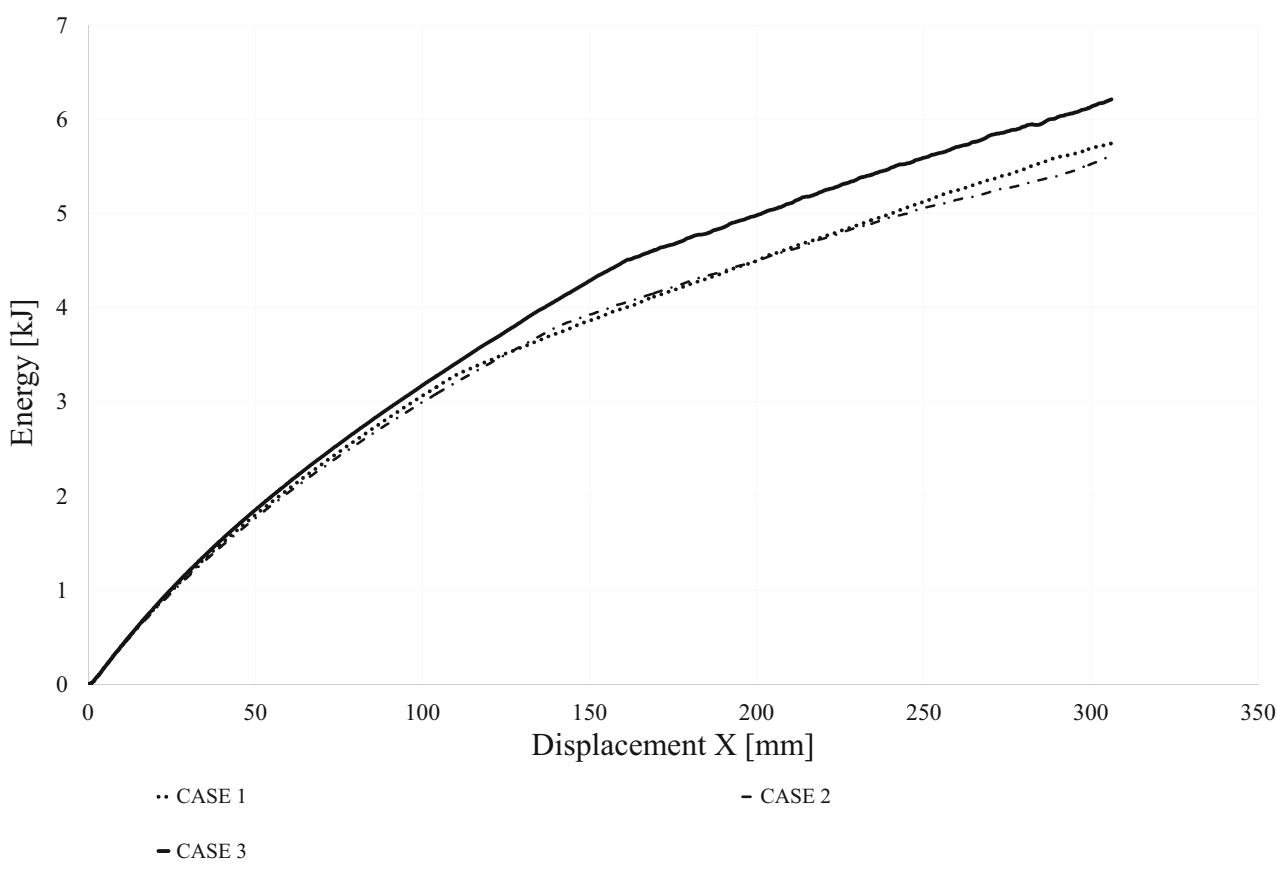




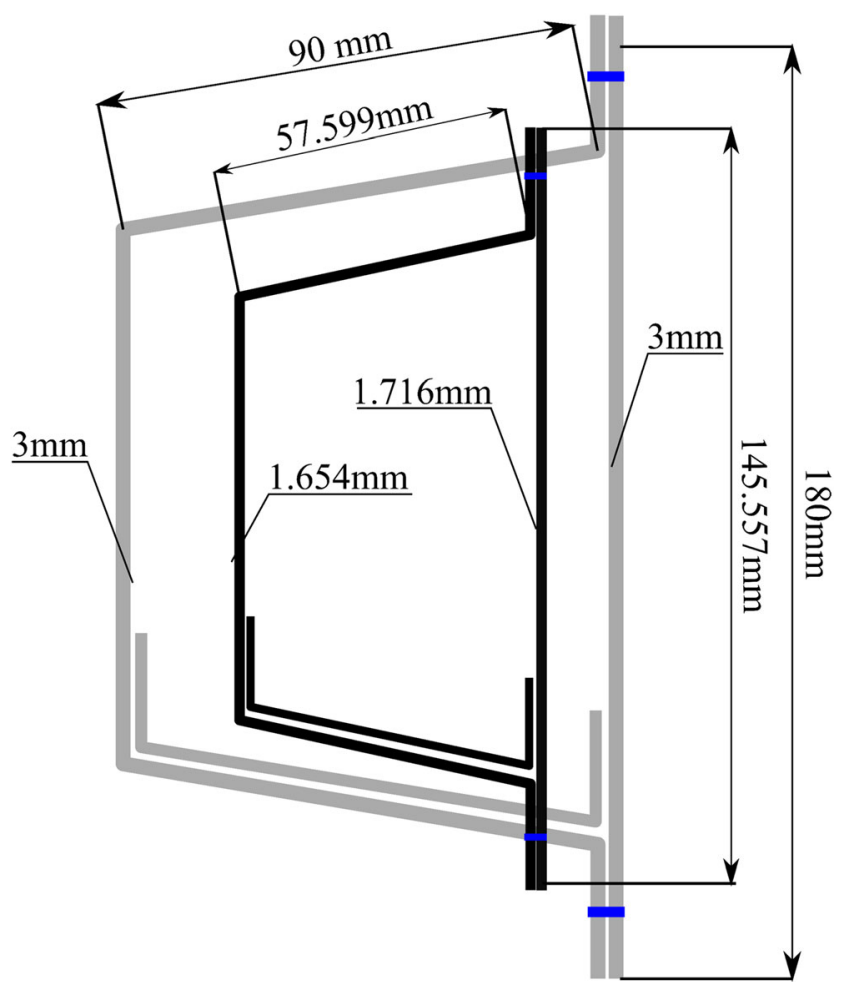

Fig. 14 Comparison between the starting estimation of the solution (gray profile) and the best result obtained in EA optimization (black profile)

dard deviations is even slightly higher than in the previous problem. The additional condition in the form of maximal moment definition had a marginal impact on algorithm results. Additionally, the solution calculated by taking the averaged

Table 1 Parameters of space frame beam model

\begin{tabular}{lll}
\hline Geometry & Parameter & Value \\
& Thickness $T 1$ & $1-3[\mathrm{~mm}]$ \\
& Length $L 1$ & $120-180[\mathrm{~mm}]$ \\
& Thickness $T 2$ & $1-3[\mathrm{~mm}]$ \\
& Length $L 2$ & $55-90[\mathrm{~mm}]$ \\
Crushing properties & Reference $S E A_{\text {ref }}$ & $1[\mathrm{~kJ} / \mathrm{kg}]$ \\
& Reference $P_{m-\text {-ref }}$ & $25,000[\mathrm{~N}]$ \\
& Reference $P_{\text {max-ref }}$ & $53,000[\mathrm{~N}]$ \\
& Reference $M_{x-\text { max-ref }}$ & $6600[\mathrm{Nm}]$ \\
& Reference $M_{y-\text { max-ref }}$ & $10,455[\mathrm{Nm}]$ \\
& L/T & $20-50$ \\
Material properties & Material density & $7900\left[\mathrm{~kg} / \mathrm{m}^{3}\right]$ \\
& Proof stress & $350[\mathrm{MPa}]$ \\
& Proof strain & $0.02 \%$ \\
& Young's modulus & $210,000[\mathrm{MPa}]$ \\
& Ultimate tensile strength & $650[\mathrm{MPa}]$ \\
\hline
\end{tabular}

Table 2 Average results of design parameters

\begin{tabular}{lcl}
\hline Variable & Average $[\mathrm{mm}]$ & Standard deviation \\
\hline Thickness $T 1$ & 1.716 & 0.098 \\
Length $L 1$ & 145.557 & 2.740 \\
Thickness $T 2$ & 1.654 & 0.092 \\
Length $L 2$ & 57.559 & 3.323 \\
\hline
\end{tabular}

values obtained in 10 independent runs indicated a small decrease of $S E A, P_{\max }$, and $P_{m}$ with respect to the previous case 1 which is also confirmed by the average fitness function results in Fig. 11. At the same time, the structural mass increased to $7.672 \mathrm{~kg}$. The dimensions of the best individual in case 2 are $L 1=142.151, L 2=61.141, T 1=2.013$, and $T 2=1.486$, expressed in millimeters. That concludes that the application of constraints (7) and (8) was not very efficient in the developed procedure.

\subsection{Final formulation of the optimization problem (case 3)}

The final calculation takes into account all formulations defined by (1)-(9). Now the results presented in Figs. 7, 8,9 , and 10 depict much better convergence than it was in two previous cases. The values of design variables are much closer to average values. Table 2 presents the average results. Now the variable $T 1$ is more than 3 times closer to average value compared with previous, under-constraint, problems. Multiple tests showed that the combination of all constraints acting together brought the desired result. The highest fitness function value in Fig. 11 is registered in the 4th run. The best SEA value $(0.816 \mathrm{~kJ} / \mathrm{kg})$ is higher in the third case than in previous two cases, the value of mean crushing force raised, which have an impact on energy absorbed by the structure. Besides, Figs. 12 and 13 present the resulting curves related to the forces and energy registered during the calculations of the best fitness function in three analysis formulations. Figure 12 shows that the peak forces for all cases are very close to the limit value. It confirms that the highest mean crushing force value was obtained for the solution which considers all conditions defined by (1)-(9). The thick solid line (case 3) is located above the remaining curves. The mean crushing force is directly related with the energy absorbed by the structure. It can be noticed in Fig. 13 that the highest amount of energy is dissipated by the deformation mechanisms corresponding to case 3 . 
Table 3 The best solutions obtained in optimization process

\begin{tabular}{llllllllllll}
\hline & $T 1[\mathrm{~mm}]$ & $L 1[\mathrm{~mm}]$ & $T 2[\mathrm{~mm}]$ & $L 2[\mathrm{~mm}]$ & $L / T$ & $P_{\max }[\mathrm{kN}]$ & $P_{m}[\mathrm{kN}]$ & Energy $[\mathrm{kJ}]$ & $S E A[\mathrm{~kJ} / \mathrm{kg}]$ & $\mathrm{Mass}[\mathrm{kg}]$ & Fitness \\
\hline Case 1 & 1.746 & 131.190 & 1.574 & 66.392 & 31.837 & 52.534 & 20.229 & 5.942 & 0.803 & 7.396 & 0.868 \\
Case 2 & 2.013 & 142.151 & 1.486 & 61.141 & 32.186 & 51.891 & 19.845 & 5.907 & 0.770 & 7.672 & 0.801 \\
Case 3 & 1.716 & 145.557 & 1.654 & 57.599 & 31.534 & 52.472 & 21.653 & 6.218 & 0.816 & 7.617 & 0.871 \\
\hline
\end{tabular}

\subsection{Summary of results}

Table 3 summarizes the best solutions obtained for three investigated formulations of the optimization problem. Each study returned different values, but main constraints were always satisfied, i.e., $P_{\max }$ and $P_{m}$ were lower than assumed limit values. Comparing the cases 1 and 2 , one can see that the slightly higher values are returned for the second case what is reflected in the fitness function value. The case 3 is clearly the best from all considered variants. The energy absorbed by the optimized structure is similar and equal to $5.942 \mathrm{~kJ}$ and $5.907 \mathrm{~kJ}$ respectively for the first and second case. However, the energy absorbed by the third case solution reached $6.218 \mathrm{~kJ}$ (Fig. 13). The presented values combined with the mass of the structure result in the lowest specific energy absorption for the second case and the highest energy absorption for the third case $(0.816 \mathrm{~kJ} / \mathrm{kg})$. It can be noticed that thickness variables returned in the first calculation are much closer to the best solution than it was in the second optimization approach. The opposite situation is visible for length variables. This suggests that the thickness variables in the considered structure have a stronger impact on the results than the length variables. The values of the relationship between lengths of cross-section side faces and their thicknesses obtained for cross sections from EA processing indicate that the constraint (6) is fulfilled for all cases. Hence, the thin-walled cross section is considered without the risk of unpredictable crushing mode (Jones and Abramowicz 1986). Additionally, Table 4 presents the cross-sectional moments in the obtained solutions and confirms the influence of the applied constraint (9) to returned solutions. The case 1 returned the most distant solution comparing to remaining solutions which are "enhanced" in some sense with the additional knowledge about the problem, included in the constraint (9). The solution which returned the highest final fitness value was obtained by the EA optimization program for the formulation that took into account all constraints. In Fig. 14, it is compared schematically to the input data starting point used for each case and defined by taking the upper limit values for all variables (Table 1 ).

\section{Conclusion and final remarks}

The investigated problem was complex and required an insight review of the initial approach to obtain consistent results. The definition of a new design criterion related to maximal bending moments in the structure was necessary to obtain stable and reliable results in several runs. The optimization problem was solved on a standard PC within a reasonably short processing time. It can be noticed that the macro element approach, applied in the VCS software, combined with the evolutionary algorithm, creates a unique solution which until now has no comprehensive coverage in the scientific literature. It constitutes a powerful tool that can process a large amount of data. The advantages of the proposed solution are evident and give the possibility of further development. Except for the growth of the structure or definition of additional variables and parameters, it is still possible to modify or enhance the optimization scheme proposed by the authors. Also, different optimization strategies can be also developed thanks to userfriendly object-oriented programing. This step is intended in the further studies.

Table 4 Cross-section moments

\begin{tabular}{lllcc}
\hline & $M y(X Z)[\mathrm{Nm}]$ positive & $M y(X Z)[\mathrm{Nm}]$ negative & $M x(Y Z)[\mathrm{Nm}]$ positive & $M x(Y Z)[\mathrm{Nm}]$ negative \\
\hline Case 1 & 6001.487 & -6710.258 & 8804.758 & -8633.107 \\
Case 2 & 5848.093 & -6531.404 & 9404.293 & $-10,058.465$ \\
Case 3 & 5478.637 & -6344.940 & $10,781.385$ & $-10,472.573$ \\
\hline
\end{tabular}


Acknowledgements Authors would like to thank Impact Design Europe company for support and express gratitude for providing indispensable tools for the research.

\section{Compliance with ethical standards}

Conflict of interest The authors declare that they have no conflict of interest.

Replication of results All necessary data of modeled structure have been given in the text of the paper (e.g., geometry of the S-frame, material properties, loading conditions). In the chapter 4.2 , the formulation of the fitness function, penalty function, and penalty coefficients were detailed. Also, the operators and parameters of the evolutionary algorithm (developed in C\#) were detailed in 4.3. Since the EA is used in the presented work, the optimization results might be verified using a similar optimization tool, but the main difficulty resides in the ability to model the crashworthiness phenomena in short processing time. The use of standard FE solvers makes the solution of the problem very hard because of time-consuming procedures related to high-speed dynamics studies. Furthermore, the AE belong to stochastic search methods and several independent runs of the optimization procedure might be necessary to check its efficacy and convergence rate for a given set of parameters.

The numerical results presented in the paper have been obtained using the software Visual Crash Studio (VCS) that applies the macro element method to model the dynamics and energy absorption of thin-walled elements. The authors had the access to this powerful tool thanks to the courtesy of the Impact Design Europe company. The VCS is a commercial tool and is not free for use. Besides, its use as a batch-mode solver needed advanced knowledge of the program structure and encoding details.

The modeling results using the VCS program of some typical problems have been validated in the past in many papers mentioned already in the chapters 3.1 and 3.2 (Takada and Abramowicz (2003); Georgiou and Zeguer (2018); Kim and Wierzbicki 2004). The validation of crashworthiness results for a thin-walled beam was presented, for example, in the previous work of authors, i.e., Pyrz and Krzywoblocki (2017).

Open Access This article is distributed under the terms of the Creative Commons Attribution 4.0 International License (http:// creativecommons.org/licenses/by/4.0/), which permits unrestricted use, distribution, and reproduction in any medium, provided you give appropriate credit to the original author(s) and the source, provide a link to the Creative Commons license, and indicate if changes were made.

\section{References}

Abramowicz W (2004) An alternative formulation of the FE method for arbitrary discrete/continuous models. Int J Impact Eng 30(8-9): 1081-1098

Ambrosio JAC (2001) Energy management and occupant protection. Springer Verlag, Wien

Cai K, Wang D (2017) Optimizing the design of automotive S-rail using grey relational analysis coupled with grey entropy measurement to improve crashworthiness. Struct Multidiscip Optim 56(6):1539 1553

Chen W, Wierzbicki T (2000) Torsional collapse of thin-walled prismatic columns. Thin-Walled Struct 36:181-196

Christensen J, Bastien C (2015) Nonlinear optimization of vehicle safety structures: Modeling of Structures Subjected to Large Deformations. Butterworth-Heimann
Christensen J, Bastien C, Blundell MV (2012) Effects of roof crush loading scenario upon body in white using topology optimization. Int $\mathbf{J}$ Crashworthiness 12(1):29-38

Chung TE, Lee YR, Kim CS, Kim HS (1996) Design of aluminum space frame for crashworthiness improvement. SAE Technical Paper 960167

De Gaetano G, Mundo D, Maletta C, Kroiss M, Cremers L (2015) Multiobjective optimization of a vehicle body by combining gradientbased methods and vehicle concept modelling. Case Stud Mech Syst Signal Process 1:1-7

Duddeck F, Hunkeler S, Lozano P, Wehrle E, Zeng D (2016) Topology optimization for crashworthiness of thin-walled structures under axial impact using hybrid cellular automata. Struct Multidiscip Optim 54(3):415-428

Eiben AE, Smith JE (2003) Introduction to evolutionary computing. Springer, Berlin

Fang J, Sun G, Qiu N, Kim NH, Li Q (2017) On design optimization for structural crashworthiness and its state of the art. Struct Multidiscip Optim 55(3):1091-1119

Georgiou G, Zeguer T (2018) On the assessment of the macro-element methodology for full vehicle crashworthiness analysis. Int $\mathbf{J}$ Crashworthiness 23(3):336-353

Hosseini-Tehrani P, Nikahd M (2006) Two materials S-frame representation for improving crashworthiness and lightening. Thin-Walled Struct 44:407-414

Impact Design Europe, Visual Crash Studio (2017) http://www. impactdesign.pl. Accessed: 5 January 2019

Jones N, Abramowicz W (1986) Dynamic progressive buckling of circular and square tubes. Int J Impact Eng 4(4):243-270

Jones N, Wierzbicki W (eds) (1983) Structural crashworthiness. Butterworth \& Company Publishers Ltd, London

Khakhali A, Nariman-zadeh N, Darvizeh A, Masoumi A, Notghi B (2010) Reliability-based robust multi-objective crashworthiness optimisation of S-shaped box beams with parametric uncertainties. J Crashworthiness 15(4):443-456

Kim HS, Wierzbicki T (2000) Numerical and analytical study on deep biaxial bending collapse of thin-walled beams. Int J Mech Sci 42(10): 1947-1970

Kim HS, Wierzbicki T (2001a) Crush behavior of thin-walled prismatic columns under combined bending and compression. Comput Struct 79:1417-1432

Kim HS, Wierzbicki T (2001b) Effect of the cross-sectional shape of hattype cross-sections on crash resistance of an "S"-frame. Thin-Walled Struct 39(7):535-554

Kim HS, Wierzbicki T (2004) Closed-form solution S-frames with rectangular cross-section. Int J Impact Eng 30(1):87-112

Liu Y (2010a) Thin-walled curved hexagonal beams in crashes-FEA and design. Int J Crashworthiness 15(2):151-159

Liu Y (2010b) Crashworthiness design of thin-walled curved beams with box and channel cross sections. Int J Crashworthiness 15(4):413423

Liu Y, Day ML (2006) Simplified modelling of thin-walled box section beam. Int J Crashworthiness 11(3):263-272

Liu Y, Day ML (2007) Development of simplified thin-walled beam models for crashworthiness analyses. Int J Crashworthiness 12(6): 597-608

Liu ST, Tong ZQ, Tang ZL, Zhang ZH (2014) Design optimization of the S-frame to improve crashworthiness. Acta Mech Sinica 30(4):589 599

Meadows D, Seeds A, McGregor I, Kenyon M (1992) Aluminum crash members in axial and bending collapse, SAE International, SAE Paper No. 922113, https://doi.org/10.4271/922113

Michalewicz Z (1996) Genetic algorithms + data structures = evolution programs, Third Revised and Extended Edition. Springer-Verlag, Berlin 
Nguyen VS, Wen G, Yin H, Van TP (2014) Optimisation design of reinforced S-shaped frame structure under axial dynamic loading. Int J Crashworthiness 19:385-393

Ni CM (1973) Impact response of curved box beam-columns with large global and local deformations. Proc. 14th Structures, Structural Dynamics, and Materials Conference. Paper No. 73-410

Ni CM (1974) A numerical method for non-linear analysis of planar frames, Proc. International Conference on Computational Methods in Non-Linear Mechanics, Austin, Texas, Sept. 23-25, 1974

Pyrz M, Krzywoblocki M (2017) Crashworthiness optimization of thinwalled tubes using macro element method and evolutionary algorithm. Thin-Walled Struct 112:12-19

Takada K, Abramowicz W (2003) Fast crash analysis of 3D beam structures based on object oriented formulation. SAE 04B-119

Wierzbicki T, Abramowicz W (1984) Dynamic axial crushing of square tubes. Int J Impact Eng 2(2):179-208
Wierzbicki T, Abramowicz W, Kecman D, Rhodes J (1989) Manual of crashworthiness engineering, vol $1-8$. MIT, Center for Transportation studies, Boston

Zhang Y, Zhu P, Chen G (2007) Lightweight design of automotive front side rail based on robust optimisation. Thin-Walled Struct 45:670 676

Zhou Y, Lan F, Chen J (2011) Crashworthiness research on S-shaped front rails made of steel-aluminum hybrid materials. Thin-Walled Struct 49:291-297

Publisher's note Springer Nature remains neutral with regard to jurisdictional claims in published maps and institutional affiliations. 